\title{
Traitement combiné de la tuberculose du larynx (bain de lumière général à la lampe à are et interrention chirurgicale locale)
}

\section{Par N. RH. Blegvad}

To cite this article: Par N. RH. Blegvad (1921) Traitement combiné de la tuberculose du larynx (bain de lumière général à la lampe à are et interrention chirurgicale locale), Acta OtoLaryngologica, 3:1, 115-125, DOI: 10.3109/00016482109121174

To link to this article: http://dx.doi.org/10.3109/00016482109121174

曲 Published online: 08 Jul 2009.

Submit your article to this journal $[\pi$ 


\title{
Traitement combiné de la tuberculose du larynx (bain de lumière général à la lampe à arc et intervention chirurgicale locale).
}

\author{
Par N. RH. BLEGVAD, \\ Oto-laryngologiste de l'Hôpital Oeresund, Copenhague.
}

Sous le nom de traitement combiné de la tuberculose du larynx, je comprends le traitement avec le bain de lumière généralisé avec la lampe à arc, associé au traitement local du larynx, en particulier la cautérisation galvanique. Les résultats de ce traitement, que je vais avoir l'honneur d'exposer, proviennent de l'hôpital d'Oeresund qui fonctionne comme hôpital des tuberculeux pour la ville de Copenhague.

La technique du traitement est la suivante:

1. Le bain de lumière généralisé à la lampe à arc est donné de la manière suivante. Le malade, complétement déshabille, est placé en position couchée devant quatre lampes à arc très puissantes (20 ampères). Dans la salle de bains de l'hôpital, il y a place pour quatre malades à la fois. On donne un bain quotidien, commençant par des séances de $1 / 4$ d'heure; la semaine suivante, on donne un bain d'une demi-heure de durée; la suivante, des bains de $3 / 4$ d'heure et enfin, après cette semaine, on administre des bains quotidiens d'une heure.

2. Il est incontestable que le bain de lumière employé seul est capable de guérir une tuberculose du larynx et bon nombre de mes malades ont été traités exclusivement par le bain de lumière. Non seulement, on voit que les ulcérations et l'oedème des régions aryténoïdiennes peuvent disparaitre, mais, dans onze cas, j'ai vu des infiltrations très considérables disparaître sans autre traite- 
ment que le bain de lumière et la cure de silence. Mais, quoique les bains de lumière constituent la partie de beaucoup la plus importante du traitement, leur action est soutenue à un degré très considérable par un traitement local énergique. Tandis que les résultats que j'obtenais autrefois par le traitement local de la tuberculose du larynx n'étaient pas particulièrement brillants, les conditions sont tout à fait modifiées depuis que les malades ont commencé à recevoir en même temps le bain de lumière. Les cautérisations galvaniques, les excisions et l'amputation de l'épiglotte paraissent être de beaucoup mieux supportées par les malades qui reçoivent les bains de lumière que par les autres; la réaction locale est minime; la cicatrisation progresse rapidement et les récidives ne sont pas aussi fréquentes. Pour les infiltrations des bandes ventriculaires, $j$ 'emploie les piqûres en profondeur avee le galvano, à la façon de Grünwald, deux à trois piqûres profondes dans chaque infiltration. Parfois les infiltrations de la région interaryténoïdienne disparaissent toutes seules sous l'action des bains de lumière; mais, pour les infiltrations plus étendues, il faut employer soit la cautérisation par piqûres en profondeur, ou l'extirpation à la curette à double tranchant, suivie de cautérisation galvanique de la plaie. Pour les ulcérations, nous employons la cautérisation galvanique en surface ou, s'il existe seulement des granulations peu profondes ou faiblement bourgeonnantes, des badigeonnages avec l'acide lactique à $100 \%$, une fois par semaine. S'il existe des ulcérations du bord de l'épiglotte avec gêne de la déglutition, on emploie l'amputation avec la guillotine d'Alexander. Dans la périchondrite des aryténoïdes, des compresses d'eau chaude sur le cou rendent souvent des services dans la dysphagie. Je n'ai jamais vu de conséquences désagréables des interventions locales, même chez des sujets en très mauvais état. Naturellement, j'essaie tout d'abord l'action que peuvent avoir les bains de lumière seuls sur la tubercùlose du larynx et ce n'est que lorsque je vois qu'ils sont sans effet ou que l'effet est trop lent que j'ai recours à l'intervention locale.

L'observation suivante mettra en lumière l'importance du traitement local: 
Marguérite K., 17 ans; entrée le 17 juin 1918. Larynx: sur le côté droit de la région interaryténoïdienne, infiltration de dimensions moyennes, saillante et ulcérée. Sur la face supérieure de la corde vocale gauche, ulcérations étendues, avec bourgeons plats. Abondante quantité de mucosités. - La malade reçoit des bains de lumière depuis le 17 juillet 1918, jusqu'au 25 mai 1919. - 16 août 1918: larynx: infiltrations et granulations diminuées; voix très enrouée. - 20 septembre: corde vocale gauche presque normale. L'infiltration de la région interarytẻnoïdienne serait plutôt plus grande. On y pratique trois piqûres très profondes au galvano. - 6 décembre: acide lactique à $100 \%$ une fois par semaine. - 14 janvier 1919: larynx: corde vocale gauche nette; la droite est un peu ronge et tuméfiée. Dans toute la région interaryténoïdienne, il existe une infiltration plane. On pratique une cautérisation galvanique étendue de cette infiltration. 21 janvier: on fait une nouvelle cautérisation galvanique de l'infiltration interarytẻnoïdienne; on n'y réussit qu'à démi. - 22 avril: l'infiltration interaryténoïdienne est presque complétement disparue. ll y a une certaine rougeur des denx cordes vocales. La voix n'est que légèrement enrouée.

La malade part alors pour le sanatorium de Vejlefjord où elle est traitée au bain de lumière, mais sans aucun traitement local actif. L'examen fait six mois après, en novembre, montre qu'il s'est produit une aggravation importante de la tuberculose laryngée, car, en particulier, l'infiltration de la région interaryténoïdienne a augmenté, do telle sorte qu'il empêche les cordes vocales de se rapprocher, ce qui fait que la voix est très enrouée.

Le silence représente un point très important du traitement local. J'ordonne à tous les malades atteints de tuberculose du larynx un silence absolu, mais ils ont la permission de parler à voix chuchotée aphone. Les résultats seraient certainement bien meilleurs si on pouvait observer le silence complet. Mais il est loin de pouvoir en être ainsi avec ce genre de clients. Il faut un grand empire sur soi-même et un degré d'éducation non moins grand pour pouvoir rester silencieux pendant six mois, peut-être même une année toute entière et il ne faut pas s'attendre à trouver cela chez nos malades.

Enfin, ce qui joue naturellement un grand rôle, c'est que ces malades reçoivent un traitement hospitalier très soigneux dans l'hôpital excellent et beau où ils sont hospitalisés. Loin de moi la pensée de méconnaître ou de nier que par le traitement hygiénique seul on puisse obtenir la guérison de la tuberculose pul- 
monaire et laryngée: mais, point n'est besoin de bien connaître la tuberculose du larynx pour savoir que malheureusement cela ne suffit pas dans la majorité des cas.

Je vais maintenant parler des cas dont il s'agit. Comme nous l'avons dit, mes résultats proviennent de l'hôpital communal de Copenhague pour les tuberculeux, où l'on traite en permanence environ 400 malades avec tuberculose de poumon, généralement en très mauvais état et parmi lesquels, naturellement, il s'en trouve un certain nombre avec tuberculose du larynx.

Sur les nouveaux malades, au nombre de 600 environ qui viennent annuellement à la clinique laryngologique, il y a à peu près 100 tuberculeux du larynx. Depuis 1917, dans la mesure où cela a été faisable, j'ai traité par le bain de lumière tous les sujets atteints de tuberculose du larynx qui pouraient supporter ce traitement. $\mathrm{Si}$, malgré cela, je n'ai traité en tout que $100 \mathrm{ma-}$ lades par les bains de lumière, la faute en revient surtout au manque de place dans l'établissement des bains, ce qui fait qu'un grand nombre de patients n'ont pu prendre les bains de lumière. On construit actuellement un nouvel établissement pour bains de lumière, de sorte qu'il est à espérer que bientôt nous pourrons traiter tous nos malades. Cependant, nous n'avons fait aucune sélection dans nos malades; nous avons traité tous ceux qui ont pu trouver de la place, même des cas en apparence désespérés. La conséquence de cela fut que quelques malades moururent pen de temps après avoir commencé le traitement, de façon qu'on ne put obtenir chez eux de grands résultats: mais, d'autre part, il arriva de temps en temps l'heureux événement que des cas, désespérés en apparence, guérirent ou furent très considérablement améliorés; j'en citerai plus loin des exemples. De sorte que je ne regrette pas d'avoir traité tous les malades, et à Y'avenir, également, je veux soumettre tous les patients à ce traitement, même si les constatations objectives font perdre presque tout . espoir. Avec ce mode de traitement, il est impossible de faire un pronostic exact; parfois on se trompe, car des cas d'apparence peu grave continuent à s'aggraver de façon constante, malgré le traitement le plus énergique, tandisqu'au contraire, dans d'autres, de tuberculoses laryngées graves guérissent compléte- 
ment, même chez des sujets avec des poumons en très mauvais état.

Pour terminer, je vais mentionner les résultats obtenus. Antérieurement j'avais publić les.premiers résultats de ce traitement (Société danoise d'oto-laryngologie, décembre 1917, compte-rendu publié dans *Ugeskrift for Laeger 1918 et dans aHospitalstidende, 1920, p. 81): pour cette raison, je me bornerai ici à fournir des remarques complémentaires.

Nous avons traité en tout 110 malades. De ce nombre, il faut cependant éliminer 24 qui furent traités pendant une période tellement courte (deux mois ou moins) qu'on ne put obtenir une modification digne d'être mentionnèe. Parmi ces cas éliminés, il y en a un certain nombre compris dans mon travail paru dans l'Hospitalstidende et chez lesquels on obtint quelque amélioration, mais qui cessèrent le traitement ou succombèrent à leur tuberculose pulmonaire, avant qu'on ait pu obtenir un résultat appréciable. Il reste donc 86 malades. Sur ce nombre, une guérison totale fut obtenue chez 26, tandis que 11 sont à peu près guéris; je veux dire par là que le larynx n'est pas complétement normal, car, il persiste, par exemple, un peu de rougeur ou un épaississement épidermisé comme reliquat d'une infiltration; mais, il ne reste plus d'ulcérations, d'oedème ou d'autre indice d'une tuberculose en activité. 22 furent très améliorés, 12 légèrement améliorés, 3 demeurèrent sans modification, tandis que, chez 12, la tuberculose laryngée progressa malgré le traitement le plus énergique.

Il y eut donc en totalité environ $30 \%$ de guérisons complètes et si l'on y compte les guérisons presque complètes, le pourcentage est de 43. Pour apprécier ce chiffre, il faut se souvenir que dans la majorité des cas, il s'agit de sujets très atteints qui souvent sont trop malades pour pouvoir être traités dans un sanatorium et chez lesquels la tuberculose du larynx est souvent très avancée quand on commence le traitement. Ainsi sur les 26 sujets guéris, il y en avait 16 chez lesquels la tuberculose pulmonaire était au III ième stade d'après le schéma de Turban; 7 étaient au

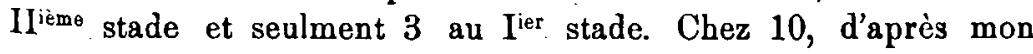
estimation, j'avais considéré la tuberculose du larynx comme 
grave, chez 6 comme de gravité moyenne et chez 10 on pouvait la considérer comme légère.

Sur les 14 malades qui sont mentionnés comme guéris dans mon travail paru dans l'Hospitalstidende, j'en ai ultérieurement examiné 7. Chẹz 5 d'entre ces derniers la guérison se main'tenait (au bout de un an à deux ans et demi); un avait eu une petite récidive qui, cependant, avait guéri complètement par la suite sous l'action du traitement à la lumière et une autre, au bout d'une année (pendant laquelle elle n'avait pas suivi le traitement par la lumière et s'était occupée de son ménage et de ses enfants) avait eu une légère atteinte de la même corde vocale qui est maintenant en traitement. 3 autres de ces malades sont morts; je n'ai pu me mettre en relation avec les 4 derniers malades. 2 des malades guéris sont morts à l'bôpital; à l'autopsie on constata que leur larynx était complètement guéri.

Je vais relater brièvement les observations des nouveaux malades guéris depuis.

Abraham David J., 27 ans. Tuberculose pulmonaire an premier. degré. 30 déeembre 1919: larynx: cordes vocales rouges. Granulations pâles sur la partie antérieure de la corde vocale droite. Sur le coté gauche de la trachée un peu au dessous de la corde vocale, une grande infiltration rouge. Voix légèrement enrouée. A reça des bains de lumière depuis le 5 janvier 1920 jusqu'à ce jour. 27 janvier 1920: larynx: l'oedème et la rougeur ont bien diminué. 10 février: il ne reste presque plus de rougeur. A peine une petite formation papillomateuse sur la partie la plus antérieure de la corde vocale droite: on l'enlève le 9 mars à la pince de Fraenkel. Le 18 mai, le larynx est complètement normal; voix claire.

Lauritz S., 43 ans. Tuberculose pulmonaire au premier degré. 14 mars 1919: larynx: partout infiltrations étendues, bourgeonnantes, surtout à droite où la bande ventriculaire et la corde vocale sont fusionnées. Voix très enrouée. Reçoit des bains de lumière depuis le 29 avril 1919 jusqu'au 6 janvier 1920. Le 29 avril, le larynx ne présente absolument aucune modification. Aphonie complète. On fait des pointes de feu profondes au galvano dans la bande ventriculaire ganche. Le 18 juin, il existe toujours de vastes infiltrations bourgeonnantes. On ordonne l'acide lactique à $100 \%$. Le 15 août, côté droit beaucoup plus détergé, cieatrisé en partie, de sorte qu'on peut faire la différenciation entre la bande ventriculaire et la corde vocale. Par contre, il existe des infiltrations étendues et ulcérées du côté gauche. 
On fait des pointes de feu profondes au galvano dans le côté gauche: 3 pointes de feu très profondes, 1 à droite, mais là on sent du tissu solide, dur, cicatriciel de sorte qu'on enlève le galvano le plus vite possible. 15 septembre: le côté gauche donne lui aussi maintenant l'impression d'etre cicatriciel, mais il est toujours quelque pen ronge. 30 novembre: partout dans le larynx, tissu cicatriciel solide. La voix est meilleure, mais elle est encore très enrouée, car la motilité des cordes vocales est encore limitée. En janvier 1920, le malade est atteint d'aliénation mentale et est transférẻ à l'Hôpital Saint-Jean, où il décède.

Laurits P. J. P., 29 ans. Tubereulose pulmonaire au troisième stade. 30 novembre 1917: larynx: petite tuméfaction pâle de la face inférieure des deux cordes vocales, s'étendant dans la trachée. 9 férrier 1918: également petite rougeur diffuse de la muqueuse. Reçoit des bains de lumière depuis le 13 septembre 1918 jusqu'au 10 décembre 1918. 8 novembre: larynx: il ne reste qu'un petit genflement pâle de la face inférieure de la corde vocale ganche; la droite parait normale. Voix légèrement enronée. 6 décembre: larynx normal. Le malade meurt le 8 janvier 1919. A l'autopsie on ne trouve pas de signe de tuberculose dans le larynx.

Johanne W., 35 ans. Tuberculose pulmonaire an troisième stade. $1^{\mathrm{er}}$ août 1919: larynx: à la partie antérieure des deux cordes vocales, ulcérations bourgeonnantes. Voix très enrouée. Reçoit des bains de lumière depuis le 11 août 1919 jusqu'au 30 janvier 1920. Le 16 septembre 1919, les granulations sont incontestablement plus petites. 7 octobre: il n'y a plus qu'un petit peu de rougeur des cordes vocales. Pas de granulations qui puissent être discernées. 22 octobre: cordes vocales maintenant presque normales; il n'y a que très peu de rougeur sur le milieu de la corde gauche. 11 novembre: cordes vocales maintenant blanches et de bel aspect: voix claire. 30 janvier 1920: larynx: état toujours normal.

Eln a Astrid R., 44 ans. Tuberenlose pulmonaire an deuxième stade. Le $1^{\text {er }}$ août 1919: larynx: corde vocale droite, rouge, gonflée, légèrement ulcérée; motilité moindre que celle de gauche. Voix légèrement voilée. 20 août: il existe du prolapsus du ventricule de Morgagnis du côté droit. Les cordes vocales sont plus ronges et plus tuméfiées. Reęoit des bains de lumière depuis le 18 octobre 1919 jusqu'au 26 mai 1920. 12 décembre: larynx: légère rougeur des deux cordes; il n'y a plus de prolapsus; pas de grannlations visibles. 5 mars 1920: il n'y a plus aucune anomalie; la voix est claire. 26 mai: élat toujours normal du larynx.

Anna P., 28 ans. Tuberculose pulmonaire au troisième stade. $1^{\text {er }}$ août 1919: larynx: fort oedème et ulcération superficielle de la 
muqueuse, surtout à la face inférieure des deux cordes vocales. Petite infiltration dans la région interaryténoïdienne. Un peu de gonflement oedémateux dans les deux régions aryténoïdiennes. Voix très enrouée. Reçoit des bains de lumière depuis le 6 août 1919 jusqu'au 7 mai 1920. 29 août: larynx: tuméfaction des régions aryténoïdiennes diminuée. 26 septembre: il n'y a plus d'oedème des régions aryténoïdiennes. Le gonflement de la face inférieure des cordes vocales est bien diminué. La surface des cordes est normale. Infiltration plane dans la région interaryténoïdienne. 21 novembre: il ne reste plus qu'une petite infiltration pâle sous la commișsure, pénétrant loin dans la profondeur de la trachée. Infiltration seulement très minime. Voix presque complètement claire. 6 février 1920: il n'y a plus qu'une petite infiltration plane non ulcérée dans la région interaryténoïdienne; les cordes vocales sont normales. 19 mars: infiltration presque entièrement disparue. 7 mai: il ne reste plus qu'une petite irrégularité plane, épidermisée de la maqueuse du côté droit de la région interaryténoïdienne.

Emma P., 44 ans. Tuberculose pulmonaire au troisième stade. 27 janvier 1920: larynx: ulcérations bourgeonnantes sur la corde vocale gauche, surtout en arrière. Rougeur et oedème de la bande ventriculaire gauche. Voix très enrouée. Reçoit des bains de lumière depuis le 4 février 1920 jusqu'à ce jour. 9 mars: corde gauche irrégulière, cicatricielle. Léger oedème; mais, pas de rougeur de la bande ventriculaire ganche. Voix claire. 20 arril: état normal. 2 juin: muqueuse partout légèrement rouge; autrement il n'y a aucune anomalie.

Carl J. A. G., 49 ans. Tuberculose pulmonaire au deuxième stade. 6 avril 1919: $\operatorname{larynx:}$ corde vocale gauche recouverte d'ulcérations bourgeonnantes très rouges. Voix voilée. Bains de lumière depuis le 14 avril 1919 jusqu'au 13 arril 1920. 2 juin: granulations diminuées. 25 août: après cocaïnisation, on constate qu'il existe une ulcération très profonde $\mathrm{de}^{*}$ la partie postérieure de la corde gauche. On fait nne cautérisation galvanique étendue de la surface de la corde vocale gauche. 23 décembre: les deux cordes vocales sont rouges; il y a toujours une ulcération sur l'apophyse vocale gauche. Voix plus claire. 23 mars: corde vocale gauche, irrégulière, rouge, mais à surface brillante, épidermisée. Voix légèrement voilée. 4 mai: il n'y a plus qu'une légère rougeur diffuse des cordes vocales. Pas d'ulcération; voix à peu près claire. 8 juin: les deux cordes vocales sont épidermisées; la voix est tout à fait claire. Le malade se sent bien et est capable de travailler;

Ruth Snefred J., 14 ans. Tuberculose pulmonaire au deuxième stade. 11 mars 1919: larynx: rougeur très forte et diffuse avec oedème de la muqueuse, surtout sur les cordes. Voix très enrouée. Pas de signe de tuberculose. 12 août: il existe des granulations sur 
les deax cordes rocales. Voix presque aphone. Bains de lumière depuis le 23 août 1919 jusqu'à ce jour. 30 septembre: granulations sur la partie antérienre de la corde gauche qui est très rouge dans sa totalité. En outre, ulcérations sur la partie antérieure des deux bandes ventriculaires. Voix très enrouée. Infiltration dans la région interaryténoïdienne. 2 décembre: il n'y a plus que des granulations pâles sur la partie antérieure des deux cordes vocales, mais, plus à gauche. Voix plus claire. 30 décembre: il n'y a plus qu'un pen de rougeur et quelques granulations sur la partie antérieure de la corde gauche, ainsi qu'une petite infiltration insignifiante dans la région interaryténoïdienne; voix presque claire. Le 26 mars, il existe sealement un pen de rougeur et de gonflement de la partie antérieure de la corde gauche. Voix claire. Rien dans la région interaryténoïdienne. 11 juin: état normal; voix très claire.

Christine Mathilde M., 23 ans. Tuberculose pulmonaire au troisième stade. 18 mars 1919: larynx: forte rougeur, mais seulement légère infiltration de la région aryténoïdienne gauche et de la région interaryténoïdienne. Petites ulcérations sur la partie postérieure de la corde ganche. Bains de lumière depuis le 25 mars jusqa'au 26 décembre 1919. 10 juin: larynx ayant meilleur aspect. $1^{\text {er }}$ août: il y a seulement un peu de rougeur, mais pas d'ulcérations visibles sur la face antérieure de la région aryténoïdienne gauche. La voix qui avait été assez enronée est maintenant presque claire. 11 juin: a été au sanatorium. Maintenant la malade vaque à ses occupations. Larynx: état complètement normal. Voix claire.

Ole Cornelius N., 28 ans. Tuberculose pulmonaire au troisième stade. 11 avril 1919: larynx: sur la corde vocale gauche, surtout en arrjère, ulcérations bourgeonnantes; voix légèrement enrouée. Bains de lumière depuis le 11 avril 1919 jusqu'au 12 décembre 1919. 9 mai: larynx: il s'eșt produite aussi une forte rougeur et, peut-être, même des ulcérations de la corde droite. Le $1^{\text {er }}$ août, il $y$ a toujours de la rougeur; mais il y a seulement des granulations sur la partie postérieure de la corde gauche. Voix toujours légèrement enrouée. Le malade sort de l'hopital le 12 décembre 1919. Il a travaillé depuis le 8 avril 1920. Il se trouve bien. 8 juin 1920: larynx: il y a seulement un peu de rougeur de la muqueuse; pas d'ulcérations; voix claire.

Betty S., 25 ans. Tuberculose pulmonaire au troisième stade. 24 janvier 1920: larynx: très fort gonflement oedémateux des deux régions arytẻnoïdiennes, surtout à gauche. Dans la région interaryténoïdienne, ulcération profonde, non détergée, qui s'étend en bas sur la paroi postérieure; en outre, en avant, infiltration plane irrégulière, ulcérée. Voix légèrement enrouée. Dysphagie considérable. Bains de lumière du 4 février 1920 jusqu'à ce jour. 4 février: comme il existe 
toujours de fortes douleurs à la déglutition surtout à gauche, on fait une injection d'alcool à $80^{\circ}$ dans le nerf laryngé supérieur gauche. 11 février: depuis l'injection d'alcool il n'y a pour ainsi dire plus de douleurs à la déglutition. Larynx: oedème assez considérable, surtout dans la région arvténoïdienne gauche; ulcérations bourgeonnantes sur les deux cordes vocales. 2 mars: oedème diminué. Grandes granulations pales sur l'ulcération de la région interaryténoïdienne. 30 mars: cordes vocales presque entièrement saines; voix plus claire. 20 avril: il n'y a plus d'oedème des régions aryténoïdiennes; cordes vocales de bon aspect, blanches. Dans la région interaryténoïdienne, une petite infiltration saillante (granulations). Le $18 \mathrm{mai}$, petites granulations dans la région interaryténoïdienne. Petite irrégularité de la corde vocale gauche. Acide lactique à $100 \%$. Le $1^{\text {er }}$ juin, il ne reste qu'une petite infiltration épidermisée dans la région interarylénoïdienne. Voix claire.

Sur les 16 malades qui, dans mon travail paru dans l'Hospitalstidende, sont cités comme étant très améliorés, 1 est maintenant totalement guéri, 2 à peu près guéris, 1 est encore en traitement avec un bon résultat, 1 autre a cessé le traitement, mais est capable de travailler et son larynx est en bon état, 1 ne s'est plus présenté pour le traitement et 8 sont morts. 3 d'entre eux étaient déjà morts au moment où le travail a paru; 5 sont décédés nltérieurement et parmi eux, 3 ont succombé à des affections intercurrentes.

Sur les 19 malades qui, dans le travail en question, étaient indiqués comme légèrement améliorés, 16 avaient cessé le traitement, soit par suite de décès (8 en tout) soit parce qu'ils ne s'étaient plus représentés pour se faire traiter. Sur les 3 autres, 1 est maintenant complètement guéri, 1 autre presque guéri, tandis que le $3^{\text {ième }}$ présenta une aggravation continue tant du côté du poumon que du côté du larynx. Il succomba six mois plus tard.

Sur les 12 malades chez lesquels la tuberculose du larynx fit des progrès incessants, presque tous étaient en très mauvais état du fait de leur tuberculose pulmonaire: 4 avaient une tuberculose laryngée grave, 5 une tuberculose du larynx de gravité moyenne et 3 une tuberculose laryngée légère.

1 de ces malades est encore en traitement; il a une tuberculose laryngée légère et est, peut-être, susceptible de.s'améliorer encore; 
2 ne se sont pas représentés pour le traitement et les 9 autres ont tous succombé à leur tuberculose pulmonaire.

Je ne puis terminer cette communication sans faire ressortir de nouveau combien il est nécessaire d'accorder une attention toute spéciale à la tuberculose du larynx. Le traitement au bain de lumière généralisé a démontré que la tuberculose du larynx, par un traitement approprié, peat guérir dans environ $30 \%$ des cas, même quand il s'agit de cas graves, chez des sujets très atteints. On peut s'attendre à ce que les résultats seront encore meilleurs si les cas se présentent de bonne heure au traitement. Il faut pour cela établir un Institut central, un sanatorium ou un hôpital où pourront être réunis tous les malades atteints de tuberculose du larynx, et où ils pourront recevoir un traitement compétent fait par un laryngologiste. Espérons qu'avee l'aide des médecins qui soignent la tuberculose nous ne tarderons pas trop longtemps à voir ce plan se réaliser, malgré la dureté des temps et les circonstances défavorables. 